\title{
Analysis of medicine consumption in peacekeeping level II hospitals
}

\author{
Lan $\mathrm{Qu}^{1,2}$, Huiling Liu ${ }^{3}$, Chunfeng $\mathrm{Li}^{4}$, Guangkai Gao ${ }^{2}$ \\ ${ }^{1}$ Jinan Military General Hospital, Jinan, China \\ ${ }^{2}$ Department of Hyperbaric Medicine, No.401 Hospital of People's Liberation Army, Qingdao, China \\ ${ }^{3}$ Department of Nephrology, No.401 Hospital of People's Liberation Army, Qingdao, China \\ ${ }^{4}$ Department of Information, No.401 Hospital of People's Liberation Army, Qingdao, China
}

\begin{abstract}
Background: The peacekeeping military units of contributing countries are unfamiliar with the conditions prevailing in foreign mission areas and therefore have difficulties with medical supplies storage.

Aim: The aim of this study is to provide reasonable and practical guidance on the maintenance of medical supplies in the peacekeeping military units of contributing countries.

Materials and methods: A total of 1,972 prescriptions were received by the pharmacy in the peacekeeping level II hospital in the Republic of Sudan from February to July of 2009 including a total of 186 drug categories and 17,713 minimum packing units. Pairwise comparison was performed using the $\chi^{2}$ test. When the total number of samples was smaller than 40 , the Fisher's exact test was adopted for pairwise comparison. Results: The majority of the consumed medicines mainly belonged to 6 categories, including specialty drugs, anti-microbial drugs, Chinese patent medicines, gastrointestinal drugs, central nervous system drugs, and drugs regulating fluids, electrolytes, and acid-base balance. Altogether, the drugs in the 6 categories accounted for $74 \%$ of all consumed medicines that were divided into a total of 20 categories.

Conclusions: Medicine consumption in peacekeeping level II hospitals is unique, therefore the drugs used in military medical facilities should be prepared according to their actual needs in the area of peacekeeping operations.
\end{abstract}

(Int Marit Health 2014; 65, 3: 131-136)

Key words: medicine consumption, peacekeeping operation, medical supplies

\section{INTRODUCTION}

The medical institutions appointed by the United Nations in each area of peacekeeping operation are categorised into four levels. According to the "Generic Guidelines for Troop Contributing Countries Deploying Military Units to the United Nations Peacekeeping Missions", the peacekeeping military units of contributing countries are responsible for the provision and maintenance of medical supplies in peacekeeping hospitals. However, the peacekeeping military units of contributing countries are unfamiliar with the harsh conditions prevailing in foreign mission areas and thus have great difficulties with medical supplies storage. The authors of this study previously worked with a medical team in a level II hospital while serving in a peacekeeping mission area in the Republic of Sudan.

The aim of the current study is to provide reasonable guidance on the medical supplies maintenance in peacekeeping military units of contributing countries based on the actual medicine consumption in level II hospitals in a mission area to better serve peacekeeping personnel and reduce medicines wastage. No similar studies have been published to date. 


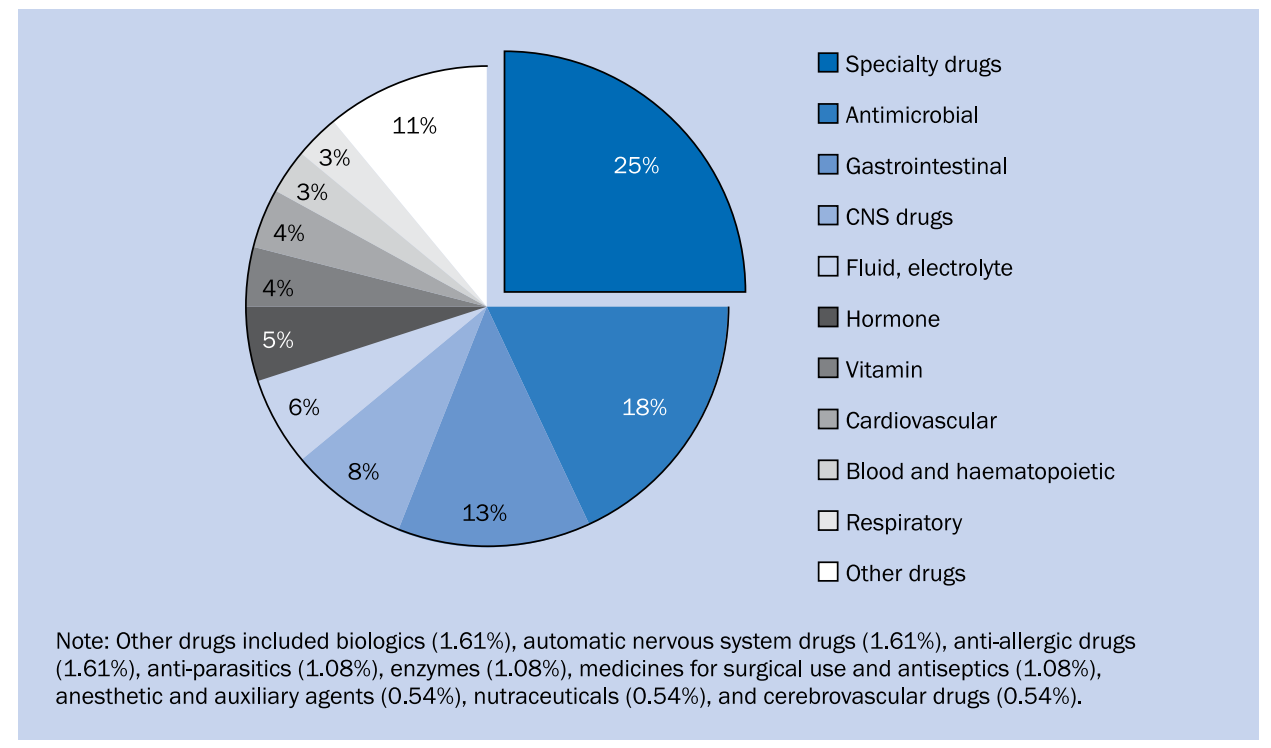

Figure 1. Proportions of each drug category; CNS - central nervous system

\section{MATERIALS AND METHODS}

The peacekeeping level II hospital in United Nations Mission in Sudan (UNMIS) Sector II in the Republic of Sudan mainly served more than 1,700 of UNMIS participants, sometimes also served local people and UNMIS participants from other Sectors. From February to July of 2009, a total of 1,328 patients were treated and overall 1,972 prescriptions were received by the pharmacy in a peacekeeping level II hospital from February to October of 2009, including a total of 186 drug categories. A total of 17,713 minimum packing units were prescribed in 14 dosage forms, including tablets, capsules, injections, creams, ointments, and drops.

All data were entered into an Excel spreadsheet for calculation. In accordance with the pharmacological classification method included in the $16^{\text {th }}$ edition of the "New Materia Medica" published by the People's Medical Publishing House [1], the 186 drugs were classified into 20 categories and 70 subcategories.

Pairwise comparison was performed using the $\chi^{2}$ test. When the total number of samples was smaller than 40 , the Fisher's exact test was adopted for pairwise comparison. The statistical software SPSS15.0 was used for the analysis.

\section{RESULTS}

\section{PROPORTIONS IN THE 20 CATEGORIES OF CONSUMED MEDICINES}

As regards the number of consumed medicines, the proportions in each of the 20 categories of consumed medicines, from highest to lowest, was as follows: specialty drugs; antimicrobial drugs; gastrointestinal drugs; central nervous system (CNS) drugs; drugs regulating fluids, electro- lytes, and acid-base balance; hormones and related drugs; vitamins; drugs acting on the cardiovascular system; drugs affecting the blood and haematopoietic system; and drugs acting on the respiratory system. Other drugs, such as biologics and drugs falling into the other 9 categories, represented a small percentage of the consumed medicines (Fig. 1).

\section{SIGNIFICANT DIFFERENCES AMONG THE 20 DRUG CATEGORIES}

Specialty drugs, antimicrobial drugs, and Chinese patent medicines ranked as the top- 2 most common drugs (the latter 2 with the same percentage), without a statistically significant difference between these 3 categories. Specialty drugs exhibited very significant differences compared to the other 17 categories of drugs, including gastrointestinal and CNS drugs. Gastrointestinal drugs ranked third, and the consumption of this category medicines was markedly higher than that of the drugs acting on the other 4 major systems, including cardiovascular and respiratory systems, with significant or very significant differences between these groups. CNS drugs ranked fourth, with significant or very significant differences compared to drugs acting on the respiratory system and anti-allergic drugs (Table 1 ).

\section{PROPORTION AND SIGNIFICANT DIFFERENCES AMONG THE 70 SUBCATEGORIES OF CONSUMED MEDICINES}

Specialty drugs. Among the specialty drugs, dermatological and ophthalmological drugs ranked first and second, accounting for $54 \%$ and $26 \%$ of the consumed medicines respectively. Otorhinolaryngological, stomatological, and gyneacological drugs tied for third place, all accounting 
Table 1. Significant differences between the 20 drug categories

\begin{tabular}{|c|c|c|c|c|c|c|c|c|c|c|c|}
\hline $\mathbf{P}\left(\chi^{2}\right)$ & \begin{tabular}{|l} 
Spe- \\
cialty \\
drugs \\
\end{tabular} & $\begin{array}{l}\text { Antimi- } \\
\text { crobial }\end{array}$ & $\begin{array}{l}\text { Gastro- } \\
\text { intesti- } \\
\text { nal }\end{array}$ & $\begin{array}{l}\text { CNS } \\
\text { drugs }\end{array}$ & $\begin{array}{l}\text { Fluid, } \\
\text { electro- } \\
\text { lyte }\end{array}$ & $\begin{array}{l}\text { Hormo- } \\
\text { ne }\end{array}$ & Vitamin & $\begin{array}{l}\text { Respi- } \\
\text { ratory }\end{array}$ & $\begin{array}{l}\text { Anti-al- } \\
\text { lergic }\end{array}$ & $\begin{array}{l}\text { Anti-pa- } \\
\text { rasitic }\end{array}$ & $\begin{array}{l}\text { Anaes- } \\
\text { thetic }\end{array}$ \\
\hline Specialty drugs & & 0.138 & $0.007 * *$ & & & & & & & & \\
\hline Antimicrobial & 2.203 & & 0.216 & $0.013 * *$ & & & & & & & \\
\hline Gastrointestinal & 7.272 & 1.531 & & 0.202 & $0.033^{*}$ & & & & & & \\
\hline CNS & & 6.168 & 1.629 & & & & 0.168 & $0.026^{*}$ & & & \\
\hline Fluid, electrolyte & & & 4.525 & & & & & & 0.078 & $0.032^{\star}$ & \\
\hline Hormone & & & & & & & & & 0.126 & $0.05^{*}$ & \\
\hline Vitamin & & & & 1.902 & & & & & & 0.092 & $0.032 *$ \\
\hline Respiratory & & & & 4.993 & & & & & & & 0.177 \\
\hline Anti-allergic & & & & & 3.100 & 2.342 & & & & & \\
\hline Anti-parasitic & & & & & 4.59 & 3.70 & 2.847 & & & & \\
\hline Anaesthetic & & & & & & & 4.600 & 1.825 & & & \\
\hline
\end{tabular}

${ }^{*} p<0.05 ; * * p<0.01$; CNS - central nervous system; the number of different drugs was the same between Chinese patent medicines and antimicrobial drugs, between vitamins and cardiovascular drugs, between drugs acting on the respiratory system and those acting on the blood and haematopoietic system, between anti-allergic drugs and biologics and autonomic nervous system drugs, between anti-parasitics and enzymes and drugs for surgical use, and between anaesthetics and cerebrovascular drugs and neutraceuticals. Therefore, one drug was selected from each category for comparison.

for $5 \%$ of the consumed medicines. Significant differences were observed in the consumption of dermatological and ophthalmological drugs $\left(\chi^{2}=6.478, p=0.020\right)$. The ophthalmological drugs also exhibited significant differences compared to the 3 types of drugs in third place, including otorhinolaryngological drugs $\left(\chi^{2}=6.303, p=0.025\right)$.

Antimicrobial drugs. Among the antimicrobial drugs, cephalosporins accounted for the largest group of consumed medicines (21\%), followed by nitroimidazoles (18\%), quinolones (14\%), macrolides (11\%), antiviral drugs (11\%), aminoglycosides (7\%), sulfonamides (7\%), and antifungal drugs (4\%). Cephalosporins exhibited no significant differences compared to antifungal drugs, which accounted for the smallest proportion $\chi^{2}=4.082, p=0.100$ ) of consumed medicines. Similarly, cephalosporins did not show significant differences in pairwise comparisons with nitroimidazoles, quinolones, macrolides, antiviral drugs, aminoglycosides, and sulfonamides.

Chinese patent medicines. Among the Chinese patent medicines, drugs for surgical use (32\%) and stomatological drugs (14\%) ranked in the top 2 subcategories of consumed medicines. (Chinese patent medicine are legally produced a kind of the Chinese drugs pharmaceutics that Chinese medicinal herb are used as raw material. It has been used repeatedly by Chinese medical scientist for thousand years and proven to be safe and effective. The preparation techniques of Chinese patent medicine are reasonable and stable and controllable.)

Gastrointestinal drugs. Among the gastrointestinal drugs, acid reducers ranked first (24\%), followed by antidiarrheals, gastrointestinal antispasmodics, antiemetics, and drugs increasing gastrointestinal mobility; these drugs tied for sec- ond place and accounted for $14 \%$ each. Mucosal protective agents, laxatives, and supplementary drugs for hepatobiliary diseases tied for third place, each accounting for $10 \%$. Pairwise comparisons among these 7 subcategories of consumed medicines showed no significant differences. For example, the comparison of the acid reducers in first place and the laxatives in third place showed $\chi^{2}=0.618$ and $p=0.697$.

CNS medicines. Among the CNS medicines, antipyretics, analgesics, and anti-inflammatory drugs accounted for nearly a half of the medicines in this category at $46 \%$. Anti-anxiety drugs got the second place at $23 \%$, and comparison between anti-anxiety drugs, antipyretics, analgesics, and anti-inflammatory drugs did not show significant differences $\left(\chi^{2}=0.650, p=0.688\right)$.

\section{PROPORTIONS AND SIGNIFICANT DIFFERENCES IN THE CONSUMED AMOUNT OF SPECIFIC DRUGS IN EACH OF THE 70 SUBCATEGORIES}

\section{Dermatological drugs in the category of specialty drugs}

Cetirizine tablets showed the largest consumption, followed by ketoconazole ointment and clotrimazole solution. A significant difference was observed between cetirizine and ketoconazole ointment $\left(\chi^{2}=36.594, p=0\right.$ ) (Fig. 2).

\section{Antimicrobial drugs}

Cephalosporins. Ceftriaxone sodium injection and cefazolin sodium injection were most commonly used, with a very significant difference compared to cefoperazonesul- 


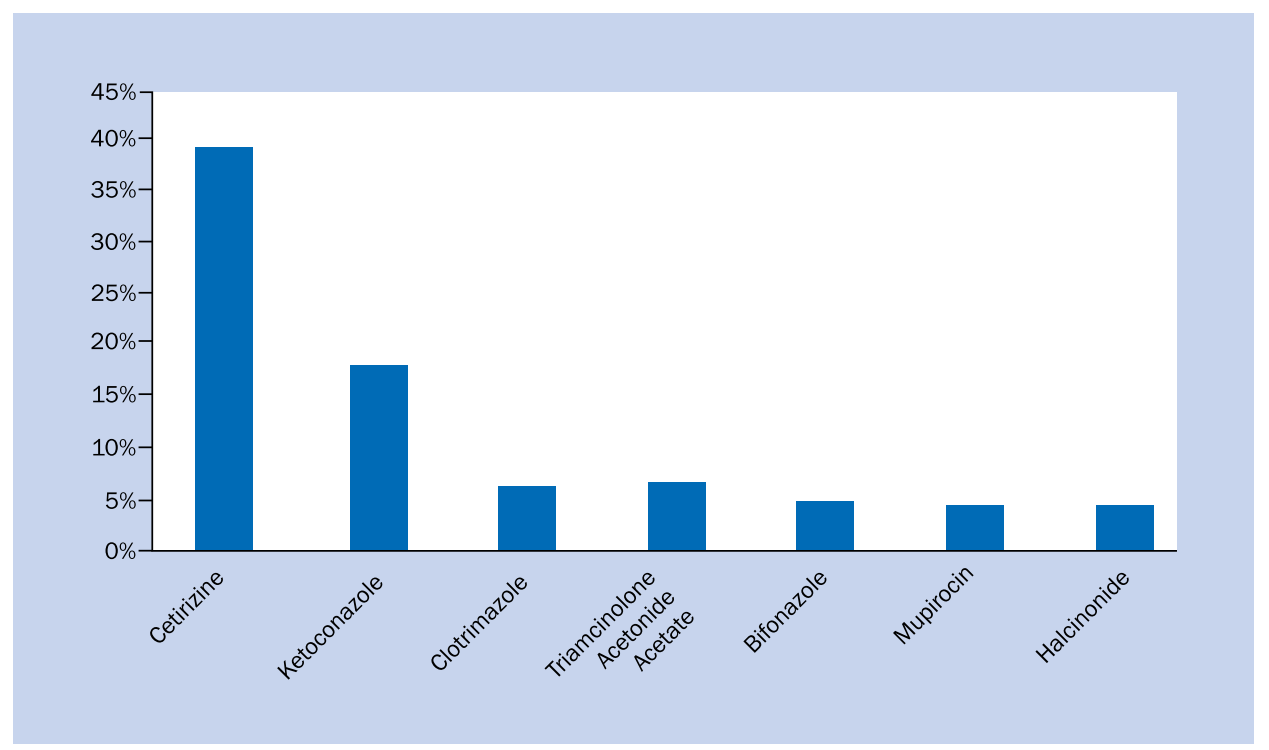

Figure 2. Dermatological drugs consumption

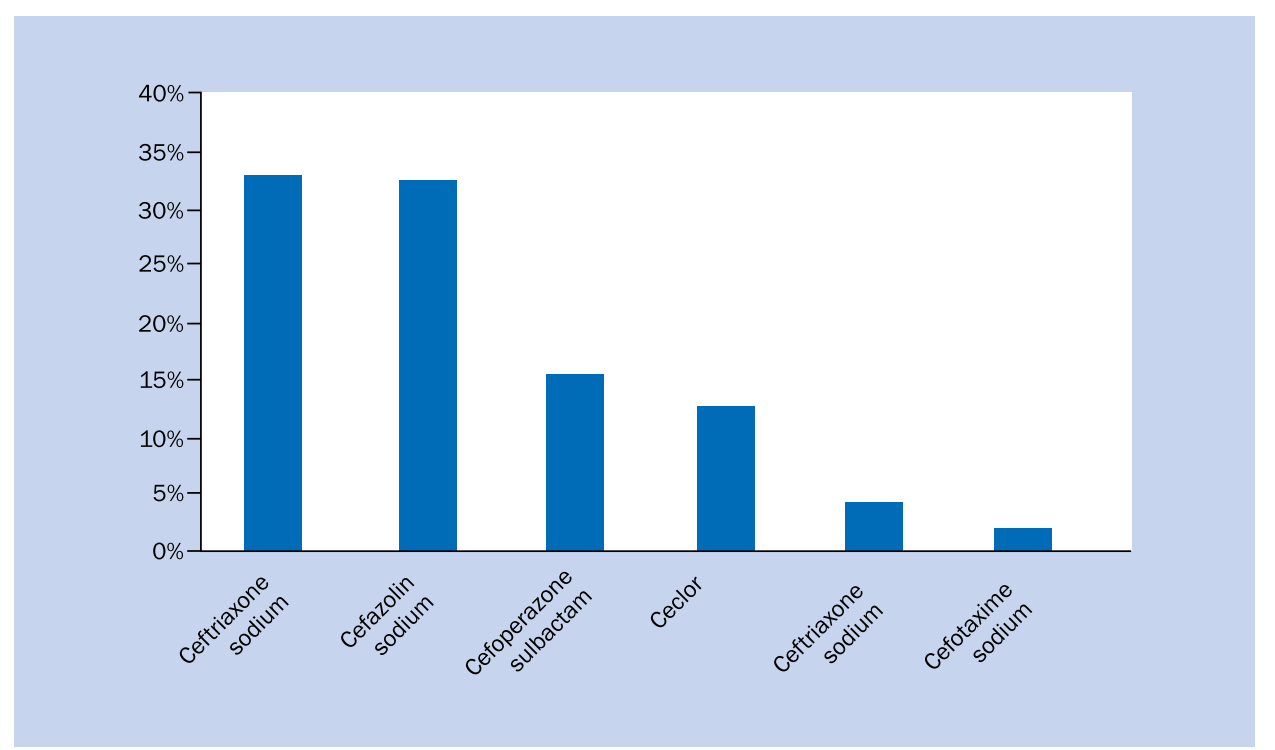

Figure 3. Antimicrobial drugs consumption

bactam injection in second place $\left(\chi^{2}=46.112, p=0\right)$. Cefoperazonesulbactam injection also showed a very significant difference in comparison to ceftriaxone sodium (Rocephin) consumption $\left(\chi^{2}=40.362, p=0\right)$ (Fig. 3).

Nitroimidazoles. Metronidazole tablets were the most commonly consumed nitroimidazole drug, with a very significant difference compared to ornidazole tablets in second place $\left(\chi^{2}=469.707, p=0\right)$. No significant difference was observed between the consumption of ornidazole tablets in the second place and tinidazole tablets in the third place $\left(\chi^{2}=1.603, p=0.229\right)$, while metronidazole injection exhibited an extremely significant difference compared to tinidazole injection $\left(\chi^{2}=34.380, p=0\right.$ ) (Fig. 4).

\section{Acid reducers in gastrointestinal drugs}

There has been a significant difference between the consumption of famotidine tablets (showing the highest consumption) and ranitidine hydrochloride capsules in second place $\left(\chi^{2}=7.923, p=0.006\right)$. However, ranitidine hydrochloride capsules showed no significant difference compared to esomeprazole enteric-coated tablets in third place $\left(\chi^{2}=1.436, p=0.259\right)$ (Fig. 5).

The most common diseases diagnosed in the peacekeeping mission area

The proportions of the most common disease diagnose in the peacekeeping mission area, from highest to low, was 


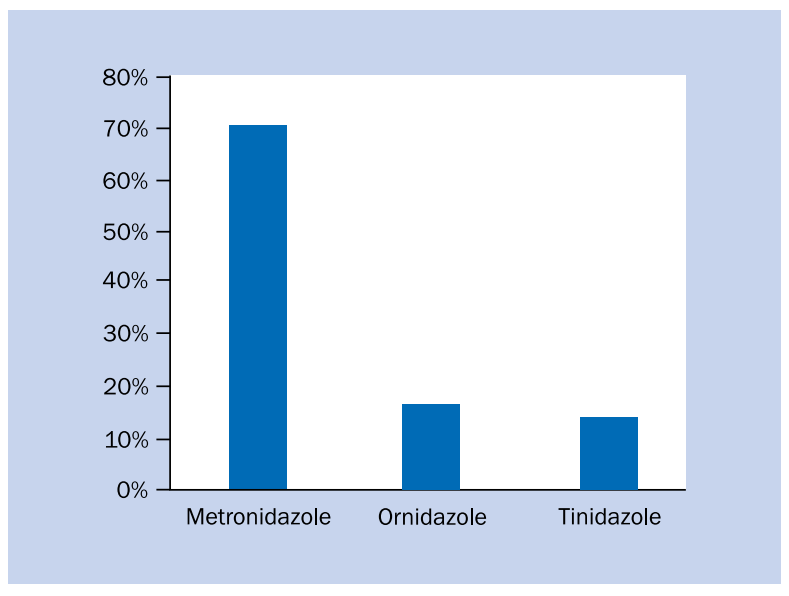

Figure 4. Nitroimidazoles drugs consumption

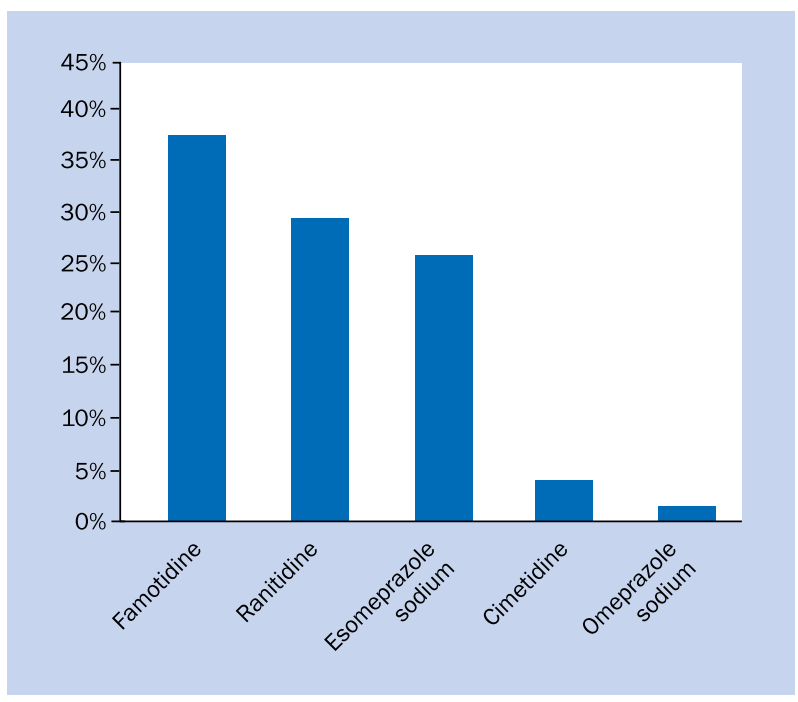

Figure 5. Acid reducers consumption

as follows: specialty disease (dermatosis, ophthalmological diseases, ear, nose and throat disease, gynaecological diseases, stomatopathy, etc.), infectious disease, traumatic disease, gastrointestinal disease, CNS disease (Fig. 6).

\section{DISCUSSION}

A total of 186 medicines were divided into 20 categories based on their pharmacological properties. The results showed that the majority of the consumed medicines mainly belonged to 6 categories, including specialty drugs, anti-microbial drugs, Chinese patent medicines, gastrointestinal drugs, CNS drugs, and drugs regulating fluids, electrolytes, and acid-base balance. All of the drugs falling into these 6 categories accounted for $74 \%$ of all consumed medicines that were divided into a total of 20 categories. The other 9 categories, including drugs such as biologics and anti-al-

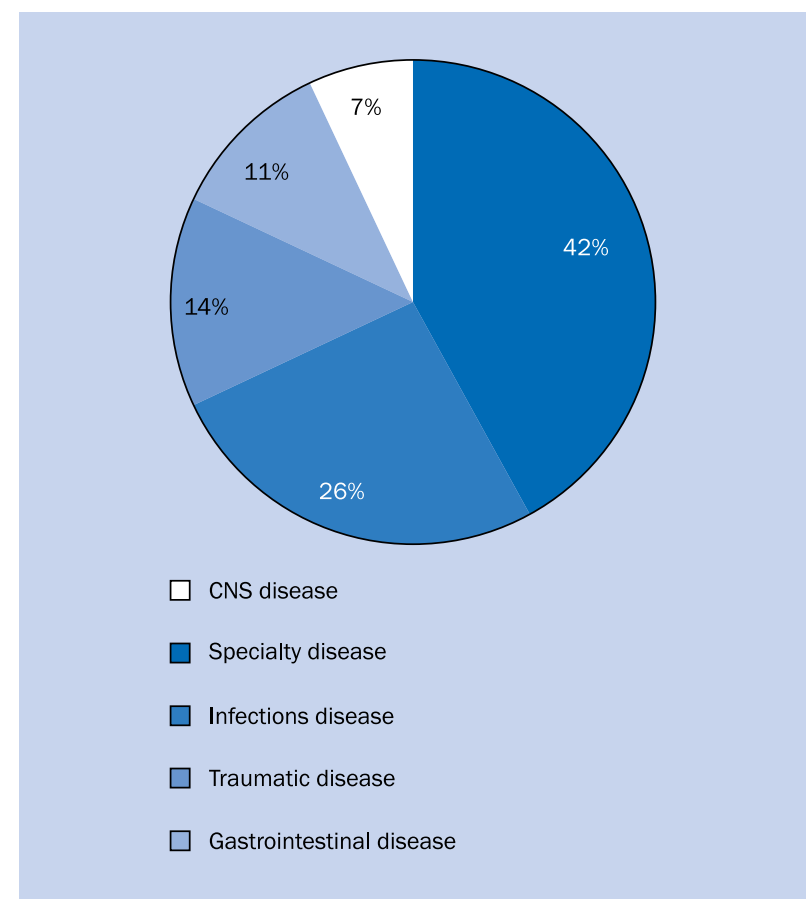

Figure 6. Proportions of the most common diseases diagnosed

lergic drugs, only accounted for a small proportion of the consumed medicines (10\%). The medicine consumption was consistent with the most common diseases diagnosed in the peacekeeping mission area.

There were 7 types of specialty drugs, including dermatological, otorhinolaryngological, and gynaecological drugs, they ranked among the most commonly consumed medicines (21\%), suggesting that the diseases of patients hospitalised in level II hospitals are different from those of typical outpatients. This difference was most likely related to the type of services provided at level II hospitals, mainly targeted at previously healthy soldiers and young people. There has been a significant increase in the use of gynaecological medicines over time, suggesting that there was an increasing number of female patients; thus, the proper provision of gynaecological medicines cannot be ignored.

Antimicrobial drugs were the second most common type of drugs, indicating that the incidence of infectious diseases remains high.

Chinese patent medicines were tied with anti-microbial drugs in the second place. Chinese patent medicines were most often used for surgical treatment, indicating a relatively high incidence of injuries.

Gastrointestinal drugs were ranked first among the 5 major system drugs, suggesting that the incidence of gastrointestinal diseases is higher than that of diseases of the other major systems. Acid reducers and antidiarrheals were the most often consumed gastrointestinal drugs, which 
could have resulted from the fact that the Sudanese diet consisted mainly of meat but lacked fruits and vegetables. In addition, the United Nations staff come from different places around the world, and the changes in the living conditions and the working environment, increased pressure, high temperature and humidity, and the consumption of perishable foods may have led to high incidence of gastrointestinal diseases, including gastritis and intestinal infection.

Among the CNS drugs, anxiolytics represented a significant proportion of the consumed medicines, suggesting that the problem of psychological stress among peacekeepers requires greater attention.

Dermatological and ophthalmological drugs were the top 2 most commonly used specialty drugs. Cetirizine tablets, ketoconazole ointment, and clotrimazole solution represented the top 3 most commonly consumed dermatological drugs, suggesting that the incidence of allergic skin diseases was the highest, followed by fungal skin infections. Norfloxacin and chloramphenicol eye drops, and erythromycin ophthalmic ointment were the top 3 consumed ophthalmic drugs, suggesting that most ophthalmic treatments targeted infections.

Cephalosporins and nitroimidazoles were the 2 most commonly consumed antimicrobial drugs. Among the cephalosporins, ceftriaxone and cefazolin sodium were consumed in the largest amounts, and metronidazole was the most consumed type of nitroimidazole drug. Cephalosporin antimicrobial drugs, especially the third-generation, have been widely used because of their broad spectrum, powerful antibacterial effects and lower level of adversity. During the 6-month study period on clinical treatment at level II hospitals, ceftriaxone was used for the treatment of 4 black and 3 white patients, and no adverse reactions were reported, suggesting that the third-generation cephalosporin drugs can be safely used in patients of different races. The choice between ceftriaxone and cefazolin sodium was mainly related to the doctors' prescription habits [2]. Between February and July of 2009, 6 cases of appendicitis were hospitalised in the level II hospital, which, together with a large number of stomatological outpatients, made the nitroimidazole drugs the second most consumed drugs.

Although the consumption of circulatory and respiratory system drugs accounted for merely $4 \%$ of the overall drug consumption, emergency drugs such as adrenaline, lobeline, remefline, and lamine must be stored, even if they not used for 6 months, as such are the requirements of a level II hospital. Antihypertensive drugs and bronchial smooth muscle spasmolytic drugs must be stored as well.

\section{CONCLUSIONS}

Drug consumption in peacekeeping level II hospitals is unique, therefore the drugs used in military medical facilities should be prepared according to their actual needs and characteristics.

Carry-on drugs should be carefully selected. The priority consideration should be placed on 6 categories of drugs, including specialty drugs, anti-microbial drugs, Chinese patent medicines, gastrointestinal and CNS drugs, as these accounted for $74 \%$ of the total medicine consumption. Of the 6 categories, dermatological drugs, cephalosporins, drugs for surgical use, antacids, antidiarrheals, antipyretics, analgesics, and anti-inflammatory drugs should be given the highest priority.

Although 9 categories of drugs, such as biologics, anti-allergy drugs, narcotic and adjuvant drugs, only accounted for a small proportion (10\%) of the consumed medicines, they should be stored in small amounts to ensure the treatment capability of peacekeeping level II hospitals and the safety of medical personnel. These medicines include tetanus antitoxin, anti-malarial drugs, and first aid and basic drugs, such as adrenaline, lobeline, remefline, and lamine.

The perishability and safety of carried drugs should also be given some consideration. More medicines should be in the form of tablets and capsules rather than explosive aerosols and perishable liquids.

The scope of treatment at peacekeeping level II hospitals should be taken into account while selecting the set of drugs to be carried into and stored in a level II medical facility. According to the "Medical Support Manual for United Nations Peacekeeping Operations", the longest duration of treatment of an inpatient admitted to a level II hospital is 7 days, and a patient must be sent to a level III peacekeeping hospital for treatment requiring longer than 7 days. Therefore, fewer long-term medications for the treatment of chronic diseases should be stored in level II hospitals. The inclusion of anxiolytics and gynaecological drugs should be considered.

\section{ACKNOWLEDGEMENTS}

This study was supported by Logistics Scientific Research Project Jinan Military Region (NO:CJN10L36).

\section{REFERENCES}

1. Liu YB, Yu Wang, Lin Hong et al. Analysis of drug application in the Wenchuan earthquake and its significance. Chinese $J$ Critical Care Med 2008; 28: 599-601.

2. Tang WB, Yan JJ. Medical quality management of Chinese peacekeeping level II hospital. Hospital Administration Journal of Chinese People's Liberation Army 2006; 13: 357-358. 